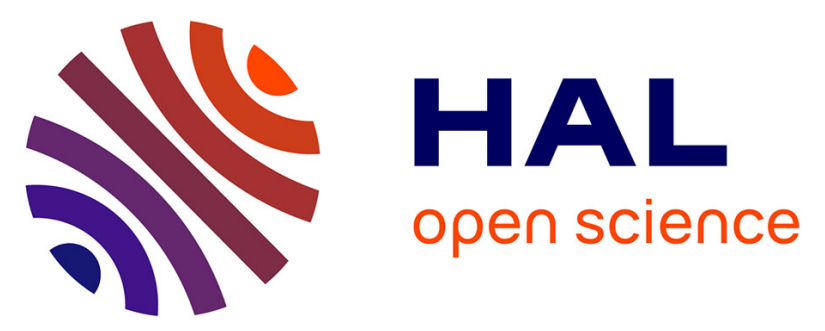

\title{
Specific versus non-specific response in exponential molecular amplification from cross-catalysis: modeling the influence of background amplifications on the analytical performances
}

Mathieu Branca, Corentin Calvet, Benoît Limoges, François Mavré

\section{To cite this version:}

Mathieu Branca, Corentin Calvet, Benoît Limoges, François Mavré. Specific versus nonspecific response in exponential molecular amplification from cross-catalysis: modeling the influence of background amplifications on the analytical performances. ChemPhysChem, 2021, 10.1002/cphc.202100342 . hal-03270075

\section{HAL Id: hal-03270075 \\ https://hal.science/hal-03270075}

Submitted on 24 Jun 2021

HAL is a multi-disciplinary open access archive for the deposit and dissemination of scientific research documents, whether they are published or not. The documents may come from teaching and research institutions in France or abroad, or from public or private research centers.
L'archive ouverte pluridisciplinaire HAL, est destinée au dépôt et à la diffusion de documents scientifiques de niveau recherche, publiés ou non, émanant des établissements d'enseignement et de recherche français ou étrangers, des laboratoires publics ou privés. 


\title{
Specific versus non-specific response in exponential molecular amplification from cross-catalysis: modeling the influence of background amplifications on the analytical performances.
}

\author{
Mathieu Branca ${ }^{[a]}$ Corentin Calvet, ${ }^{[a]}$ Benoît Limoges ${ }^{[a]}$ and François Mavré ${ }^{\star[a]}$ \\ In Memory of Pr. Jean-Michel Savéant (1933-2020)
}

[a] Dr. Mathieu Branca, Corentin Calvet, Dr. Benoît Limoges and Dr. François Mavré

Université de Paris, Laboratoire d'Electrochimie Moléculaire, UMR 7591, CNRS, F-75013 Paris, France

E-mail: francois.mavre@u-paris.fr

\begin{abstract}
Molecular based signal amplifications relying on an autocatalytic process may represent an ideal strategy for the development of ultrasensitive analytical or bioanalytical assays, the main reason being the exponential nature of the amplification. However, to take fully advantage of such amplification rates, high stability of the starting co-reactants is required in order to avoid any undesirable background amplification. Here, on the basis of a simple kinetic model of cross-catalysis including a certain degree of intrinsic instability of co-reactants, we highlight the key parameters governing the analytical response of the system and discuss the analytical performances that are expected from a given kinetic set. In particular, we show how the detection limit is directly related to the relative instability of reactants within each catalytic loop. The model is validated with an experimental dataset and is intended to serve as a guide in the design and optimization of autocatalytic molecularbased amplification systems with improved analytical performances.
\end{abstract}

\section{Introduction}

The ability of a given molecule to trigger a chemical process is at the heart of chemical or biochemical signaling and sensing. Molecular amplification occurs as soon as the triggered chemical process leads to a significantly greater amount of molecular output than the molecular input. That way, the analytical information (or signal) is amplified and therefore more readily processed, opening routes for sensitive detection or sensing..$^{[1,2]}$ In many approaches, molecular or signal amplification results from catalytic amplifications, wherein a given molecule is able to trigger the catalysis of a reaction (the given molecule being either the catalyst itself or an activator, Scheme 1). In that case, as long as the catalyst is not degraded, the product is linearly accumulated with time and the amplification factor depends only on the catalytic rate and reaction time. A typical example is the signal amplification stemming from enzyme catalysis in enzymelinked immunosorbent assay (ELISA). ${ }^{[3,4]}$

However, greater amplification rates can be achieved by introducing non-linearity in the amplification. A simple process providing non-linear amplification rates involves the coupling between two catalysts ("catalysis-to-catalysis"), where one catalyst catalyzes the production of another catalyst (Scheme 1). In such situation, the product of the second catalysis accumulates quadratically with time. Many examples of catalysis-to-catalysis have been reported so far, showing enhanced analytical performances. ${ }^{[1,2,5,6,7]}$

An even more interesting case is encountered when the molecular input initiates self-replication or autocatalysis (i.e., a process in which the trigger is not only a catalyst but also the product of it). ${ }^{[8,9]}$ Several types of autocatalysis exists, along with a wide range of mechanisms. ${ }^{[9,10]}$ Phenomenological, mechanistic or mathematical definitions have been proposed and the classification is complex and still under debate in the literature. ${ }^{[11,12,13]}$ A widely encountered model is autocatalysis based on template replication, which can be, in its most aggregated form, described as $\mathrm{X} \rightarrow 2 \mathrm{X}$, where $\mathrm{X}$ is therefore an

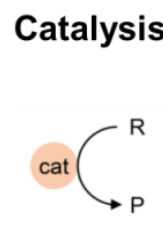

Linear amplification

Cross-catalysis

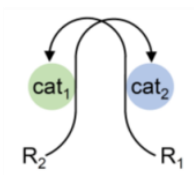

Exponential amplification

\section{Catalysis-to-catalysis}

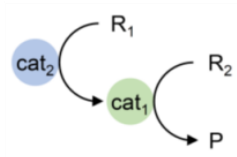

Quadratic amplification
Scheme 1. Linear and non-linear molecular-based signal amplification schemes involving catalysis. Catalysis entry: Cat catalyzes the transformation of $R$ into $P$, and $P$ is then linearly accumulated with time (amplification factor = $[P] /[C a t])$. Catalysis-to-catalysis entry: Cat 2 catalyzes the transformation of $R_{1}$ into $\mathrm{Cat}_{1}$, while $\mathrm{Cat}_{1}$ catalyzes the transformation of $\mathrm{R}_{2}$ into $\mathrm{P}$, leading to the quadratic accumulation of $P$ with time (amplification factor $\left.=[P] /\left[\mathrm{Cat}_{2}\right]\right)$. Crosscatalysis entry: Cat 2 catalyzes the transformation of $\mathrm{R}_{1}$ into $\mathrm{Cat}_{1}$, while $\mathrm{Cat}_{1}$ catalyzes the transformation of $\mathrm{R}_{2}$ into $\mathrm{Cat}_{2}$, leading to the exponential accumulation of $\mathrm{Cat}_{1}$ and $\mathrm{Cat}_{2}$ with time (amplification factor $=\left[\mathrm{Cat}_{2}\right] /\left[\mathrm{Cat}_{2}\right]_{0}$ or $\left.\left[\mathrm{Cat}_{1}\right] /\left[\mathrm{Cat}_{1}\right]_{0}\right)$. Catalysis-to-cross-catalysis entry: Cat catalyzes the transformation of $S$ or $R_{1}$ into $\mathrm{Cat}_{1}$, then $\mathrm{Cat}_{1}$ catalyzes the transformation of $R_{2}$ into $C_{2} t_{2}$ which catalyzes the transformation of $R_{1}$ into Cat ${ }_{1}$. This process finally leads to the exponential accumulation of $\mathrm{Cat}_{1}$ and $\mathrm{Cat}_{2}$ with time (amplification factor $=\left[\mathrm{Cat}_{1}\right] /[$ Cat $]$.or $\left.\left[\mathrm{Cat}_{2}\right] /[\mathrm{Cat}]\right)$.

autocatalyst. But autocatalysis may also rise from more collective behaviors such as in cross-catalysis. ${ }^{[8,12]}$ Basically, cross-catalysis involves two catalytic loops that are in a selfactivation dependency (Scheme 1), making it possible to amplify exponentially the concentration of each of the catalysts. ${ }^{[14]}$ In that respect, signal amplification based on an autocatalysis, due to its exponential nature, has certainly proved to be among the most powerful approaches for improving the analytical performances of bioassays. It is particularly true for the detection 
of nucleic acids, for which numerous strategies based on template replication have been developed. A prototypical example is the well-known Polymerase Chain Reaction (PCR), ${ }^{[15]}$ but isothermal alternatives are now widely developed, among which Exponential Amplification Reaction (EXPAR) is particularly efficient. ${ }^{[16-20]}$ For non-nucleic acid targets, it is possible to combine the high amplification power of PCR or EXPAR with the specificity of immunoassays, such as in immuno-PCR ${ }^{[21,22]}$ or immuno-EXPAR. ${ }^{[23,24]}$ However, alternatives to nucleic acid-based exponential amplification are very rare and it is a problem for target lacking immunogenicity such as small molecules. An elegant solution is the use of dendritic chain reactions, in which the target-triggered decomposition of a molecular probe leads to the release of several equivalents of molecular targets, as exemplified for the detection of fluoride anions, $\mathrm{H}_{2} \mathrm{O}_{2}$ or thiols. ${ }^{[25-27]}$ Alternatively, we recently proposed a novel autocatalytic reaction scheme that is based on cross-catalysis. ${ }^{[28]}$

Despite the power of exponential amplification rates from autocatalysis, the associated analytical performances may be quite often limited due to background amplification or nonspecific response. ${ }^{[17,18,27,28]}$ One of the major reason is the intrinsic lack of stability of the starting co-reactants, which can lead to unwanted initiation of the amplification process (therefore leading to a detrimental nonspecific signal). In other words, highly efficient molecular amplification systems are "victims" of their power, i.e. the higher the amplification of a specific input, the higher the undesired background amplification. Consequently, a critical challenge is to improve the detection sensitivity by designing systems with increased chemical stability of co-reactants while maintaining their rapid and selective reactivity. The questions we would like to address here are: (i) what degree of stability is required to lead to a sufficiently low limit of detection (LOD), and (ii) does increasing the reactivity of the system necessarily lead to better analytical performances? The ambition of the present paper is therefore to derive analytical solutions for a basic autocatalytic reaction scheme involving cross-catalysis and intrinsic instabilities of the starting co-reactants, in order to guide the design of analytically performant cross-catalytic systems for signal amplification.

Firstly, we will highlight the key parameters that govern the specific and nonspecific responses of the system and discuss the analytical performances that can be expected from a given kinetic characteristics set. Then, the model will be validated with an experimental dataset based on our previously reported autocatalytic system, ${ }^{[28]}$ involving cross-activation between $\mathrm{H}_{2} \mathrm{O}_{2-}$ mediated catalytic deprotection of a masked quinone-based probe and a quinone-redox-cycling-based catalytic $\mathrm{H}_{2} \mathrm{O}_{2}$ production.

\section{Results and Discussion}

\section{Model description and set of dimensionless differential equations}

As mentioned earlier, cross-catalysis involves two catalytic loops that self-activate through the reciprocal catalytic production of each loop's catalyst. ${ }^{[8,12,14]}$ This results in the exponential amplification of the concentration of each of the catalysts, as evidenced by Hinshelwood in its seminal paper from 1952. ${ }^{[14]}$ The simplest way to describe a cross catalytic system involves two reservoirs $\left(R_{1}, R_{2}\right)$ and two catalysts $\left(C_{1}, C_{2}\right)$. On the one hand, $C_{2}$ is catalytically produced from $R_{2}, C_{1}$ being the catalyst of loop 1 . On the other hand, $C_{1}$ is catalytically produced from $R_{1}$, $\mathrm{C}_{2}$ being the catalyst of loop 2 .

$$
\begin{array}{ll}
\mathrm{R}_{2} \underset{\mathrm{C}_{1}}{\stackrel{\mathrm{C}_{1}}{\longrightarrow}} \mathrm{C}_{2} \quad \text { (catalytic loop 1) } \\
\mathrm{R}_{1} \underset{\mathrm{C}_{2}}{\stackrel{\mathrm{C}_{2}}{\longrightarrow}} \mathrm{C}_{1} \quad \text { (catalytic loop 2) }
\end{array}
$$

In order to account for background amplification, this system of two interconnected reactions is completed by introducing the undesirable non-catalyzed reactions (leaks or instabilities), i.e. the slow first-order production of $C_{1}$ and $C_{2}$ from $R_{1}$ and $R_{2}$ in the absence of catalyst, respectively.

$$
\begin{aligned}
& \mathrm{R}_{2} \stackrel{k_{3}}{\longrightarrow} \mathrm{C}_{2} \\
& \mathrm{R}_{1} \stackrel{k_{4}}{\longrightarrow} \mathrm{C}_{1}
\end{aligned}
$$

The temporal dynamics of this system can be described by the following set of ordinary differential equations (ODE), considering the catalytic rates are first-order towards both catalysts and reservoirs, while the uncatalyzed reactions are just first-order towards reservoirs.

$$
\begin{aligned}
& \frac{d\left[\mathrm{C}_{1}\right]}{d t}=k_{2}\left[\mathrm{R}_{1}\right]\left[\mathrm{C}_{2}\right]+k_{4}\left[\mathrm{R}_{1}\right] \\
& \frac{d\left[\mathrm{C}_{2}\right]}{d t}=k_{1}\left[\mathrm{R}_{2}\right]\left[\mathrm{C}_{1}\right]+k_{3}\left[\mathrm{R}_{2}\right] \\
& \frac{d\left[\mathrm{R}_{1}\right]}{d t}=-k_{2}\left[\mathrm{R}_{1}\right]\left[\mathrm{C}_{2}\right]-k_{4}\left[\mathrm{R}_{1}\right] \\
& \frac{d\left[\mathrm{R}_{2}\right]}{d t}=-k_{1}\left[\mathrm{R}_{2}\right]\left[\mathrm{C}_{1}\right]-k_{3}\left[\mathrm{R}_{2}\right]
\end{aligned}
$$

In order to derive analytical solutions, an additional assumption must be stated. In the early stages of the reaction (induction period), the reservoirs concentrations are considered as constant. It follows that the catalyst concentrations are solutions of this simple set of ODE.

$$
\begin{aligned}
& \frac{d\left[\mathrm{C}_{1}\right]}{d t}=k_{2}^{\prime}\left[\mathrm{C}_{2}\right]+k_{4}^{\prime} \quad k_{1}^{\prime}=k_{1}\left[\mathrm{R}_{2}\right]_{0} \\
& \begin{array}{lll}
d t & \text { with } \quad & k_{2}^{\prime}=k_{2}\left[\mathrm{R}_{1}\right]_{0} \\
\frac{d\left[\mathrm{C}_{2}\right]}{d t}=k_{1}^{\prime}\left[\mathrm{C}_{1}\right]+k_{3}^{\prime} & & k_{3}^{\prime}=k_{3}\left[\mathrm{R}_{2}\right]_{0} \\
& & k_{4}^{\prime}=k_{4}\left[\mathrm{R}_{1}\right]
\end{array}
\end{aligned}
$$

The two above equations may be rearranged in a particularly simple form as a couple of dimensionless ODEs

$$
\begin{aligned}
& \frac{d^{2} c_{1}}{d \theta^{2}}-c_{1}=\alpha \\
& \frac{d^{2} c_{2}}{d \theta^{2}}-c_{2}=\beta
\end{aligned}
$$

with:

$\theta=\sqrt{k_{2}^{\prime} k_{1}^{\prime}} t ; c_{i}=\frac{\left[\mathrm{C}_{\mathrm{i}}\right]}{\left[\mathrm{R}_{1}\right]_{0}} ; \alpha=\frac{k_{3}^{\prime}}{k_{1}^{\prime}\left[\mathrm{R}_{1}\right]_{0}} ; \beta=\frac{k_{4}^{\prime}}{k_{2}^{\prime}\left[\mathrm{R}_{1}\right]_{0}}$

$\theta$ is a parameter representing the dimensionless time. It corresponds to the ratio between the reaction time $t$ and a 
characteristic time $t_{s}=1 / \sqrt{k_{1}^{\prime} k_{2}^{\prime}}$ associated to the reciprocal of the geometric mean of each loop's rate or autocatalytic efficiency. The concentration $\left[\mathrm{C}_{\mathrm{i}}\right]$ chosen to define the dimensionless concentration parameter can be indifferently selected in a range from the starting concentration in the reservoir to an arbitrary value greater than zero. Ideally, it must be an experimental observable of the system so that it allows determining the progress of the reaction and/or the half-reaction time (in the present case $\left[R_{1}\right]_{0}$ has been selected as a reference reservoir, and when the latter is half-empty it leads to the halfreaction time definition $t_{c}$ or $t_{1 / 2}$, which in a dimensionless form is given by $\left.c_{1}\left(\theta_{C}\right)=1 / 2+c_{1}^{0}\right)$. Finally, $\alpha$ and $\beta$ are parameters expressing the dimensionless ratio of the rate of non-catalyzed to catalyzed production for each catalyst, $\mathrm{C}_{2}$ and $\mathrm{C}_{1}$ respectively, which we can define as the relative instability associated with each loop.

\section{Direct triggering of the autocatalytic reaction by one of the two catalyst}

Resolution of the above system depends on the initial conditions (initial concentration of each catalyst). Two different sets of initial conditions can be envisioned, each one corresponding to each catalyst as trigger of the overall system:

(i) $\mathrm{C}_{1}$ is the trigger, that is at $\mathrm{t}=0, c_{1}^{0} \neq 0$ and $c_{2}^{0}=0$.

(ii) $\mathrm{C}_{2}$ is the trigger, that is at $\mathrm{t}=0, c_{1}^{0}=0$ and $c_{2}^{0} \neq 0$.

Additionally, the following dimensionless parameter $\gamma$ can be introduced:

$$
\gamma=\frac{k_{2}^{\prime}}{k_{1}^{\prime}}
$$

This is a competitive kinetic parameter describing the relative velocity of loop 2 with respect to loop 1 .

\section{(i) $C_{1}$ is the trigger}

Solving the system of ODEs for $c_{1}^{0} \neq 0$ and $c_{2}^{0}=0$ allows for the determination of both $c_{1}$ and $c_{2}$ as a function of $\theta$ as follows:

$$
\begin{aligned}
& c_{1}=c_{1}^{0} \cosh (\theta)+\alpha(\cosh (\theta)-1)+\sqrt{\gamma} \beta \sinh (\theta) \\
& c_{2}=\frac{c_{1}^{0}}{\sqrt{\gamma}} \sinh (\theta)+\frac{\alpha}{\sqrt{\gamma}} \sinh (\theta)+\beta(\cosh (\theta)-1)
\end{aligned}
$$

These two expressions reflect the non-linear accumulation of both catalysts with time as well as the contribution of both instabilities in the process. Note that these expressions are in line with results from Hinshelwood in the specific case where $\alpha$ and $\beta$ are equal to zero. ${ }^{[14]}$ In addition, we can deduce the evolution of the reservoirs $r_{1}$ and $r_{2}$ since:

$\frac{d r_{1}}{d \theta}=-\frac{d c_{1}}{d \theta} \quad$ and $\quad \frac{d r_{2}}{d \theta}=-\frac{d c_{2}}{d \theta}$.

After integration,

$$
r_{1}=1-\left(c_{1}-c_{1}^{0}\right)
$$

and

$$
r_{2}=r_{2}^{0}-c_{2}
$$

The agreement between the approximated analytical solutions and numerical simulation is illustrated in Figure 1 representing the evolution of both $c_{1}$ and $r_{1}$ with $\theta$. The analytical solutions derived here perfectly agree with the numerical simulations at low $\theta$ values, and so allow for prediction of the extent of the induction phase. Nevertheless, as expected, a deviation is observed as soon as the assumption of a constant concentration for the reservoirs is no longer valid.

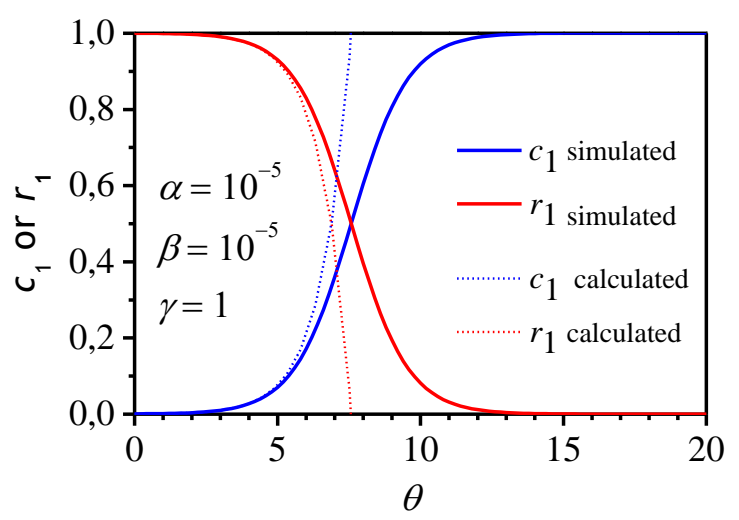

Figure 1. Evolution of $c_{1}$ or $r_{1}$ with $\theta$. Dashed lines: plots of eqs. 1 (blue) and 3 (red), respectively. Parameters set: $\alpha=10^{-5}, \beta=10^{-5}, \gamma=1, c_{1}^{0}=10^{-3}$. Plain lines: Simulated values of $c_{1}$ and $r_{1}$ using COPASI software with $k_{1}=k_{2}=1 \mathrm{M}$ ${ }^{1} \cdot \mathrm{s}^{-1}, k_{3}=k_{4}=10^{-5} \cdot \mathrm{s}^{-1},\left[\mathrm{C}_{1}\right]_{0}=10^{-3} \mathrm{M},\left[\mathrm{R}_{1}\right]_{0}=\left[\mathrm{R}_{2}\right]_{0}=1 \mathrm{M}$.
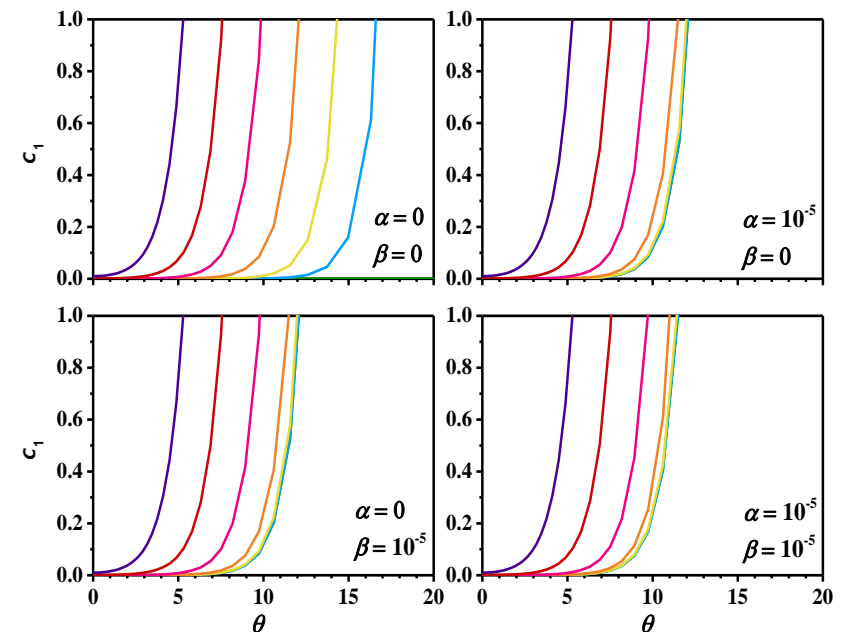

Figure 2. Evolution of $c_{1}$ with $\theta$ for different initial triggering concentrations $c_{1}^{0}$ : 0 (green), $10^{-7}$ (cyan), $10^{-6}$ (yellow), $10^{-5}$ (orange), $10^{-4}$ (pink), $10^{-3}$ (red), $10^{-2}$ (violet). The different curves were obtained from the plot of eq. 1 using the following set of parameters: $\alpha=0, \beta=0, \gamma=1$ (panel top left); $\alpha=10^{-5}, \beta=0, \gamma$ $=1$ (panel top right); $\alpha=0, \beta=10^{-5}, \gamma=1$ (panel bottom left); $\alpha=10^{-5}, \beta=10^{-5}$, $\gamma=1$ (panel bottom right).

Figure 2 depicts the evolution of $c_{1}$ with $\theta$ for various initial value of $c_{1}^{0}$ (triggering concentration) and various values of $\alpha$ and $\beta$. In all cases the symmetry factor $\gamma$ is 1 , meaning the two loops are equally fast. The panel top left (absence of instabilities, i.e. $\alpha$ 
and $\beta=0$ ) clearly shows that the catalyst concentration exponentially grows when $c_{1}^{0} \neq 0$, with decreasing induction time upon increasing the starting concentration, while in the absence of trigger $\left(c_{1}^{0}=0\right)$, it remains zero (no background amplification). In the presence of a leak (other panels), the concentration of $c_{1}$ exponentially grows even in the absence of trigger (background amplification), no matter the origin of instability. Consequently, it makes it impossible to distinguish between specific and nonspecific amplifications for the lowest triggering concentrations. Here, note that the contributions of either $R_{1}$ or $\mathrm{R}_{2}$ instability on background amplification are clearly equivalent, which is the consequence of symmetry factor $\gamma=1$.

The analytical performances of the system can be grasped by plotting the calibration curve that we have here extracted from the half-reaction time $\theta_{C}$ as a dimensionless observable (defined in our model as $r_{1}\left(\theta_{C}\right)=1 / 2$ or $c_{1}\left(\theta_{C}\right)=1 / 2+c_{1}^{0}$, which is arbitrary because many other reaction times would be also used to plot the calibration curve such as for instance $\theta_{10}$ defined as $r_{1}\left(\theta_{10}\right)=9 / 10$ or $\left.c_{1}\left(\theta_{10}\right)=1 / 10+c_{1}^{0}\right)$. In order to evaluate the influence of the different parameters on the half-reaction time, we additionally derived the following expressions for $\theta_{C}$ :

if $c_{1}^{0}+\alpha>|\sqrt{\gamma} \beta|$,

$\theta_{C}=\cosh ^{-1}\left(\frac{1 / 2+c_{1}^{0}+\alpha}{\sqrt{\left(c_{1}^{0}+\alpha\right)^{2}-\gamma \beta^{2}}}\right)-\tanh ^{-1}\left(\frac{\sqrt{\gamma} \beta}{c_{1}^{0}+\alpha}\right)$

if $\sqrt{\gamma} \beta>\left|c_{1}^{0}+\alpha\right|$,

$\theta_{C}=\sinh ^{-1}\left(\frac{1 / 2+c_{1}^{0}+\alpha}{\sqrt{\gamma \beta^{2}-\left(c_{1}^{0}+\alpha\right)^{2}}}\right)-\tanh ^{-1}\left(\frac{c_{1}^{0}+\alpha}{\sqrt{\gamma} \beta}\right)$

if $c_{1}^{0}+\alpha=\sqrt{\gamma} \beta$,

$\theta_{C}=\ln \left(\frac{1 / 2+c_{1}^{0}+\alpha}{\left(c_{1}^{0}+\alpha\right)}\right)$

Comparison of the analytically calculated and numerically simulated half-reaction times for a set of $c_{1}^{0}$ triggering concentrations (Figure 3 ) indicates that the half-reaction times can be acceptably estimated from the analytical solutions, leading to a reasonably good prediction of the calibration curve for a given kinetic set. For the highest $c_{1}^{0}$ values, the resulting calibration plot shows as expected linearity as a function of the logarithmic concentration of trigger. However at the lowest concentrations it levels off as $c_{1}^{0}$ decrease due to the limitation by nonspecific background amplification, the limiting values of which being given by the following relationships:

$$
\begin{aligned}
& \text { If } \alpha>|\sqrt{\gamma} \beta|, \theta_{C, \text { lim }}=\cosh ^{-1}\left(\frac{1 / 2+\alpha}{\sqrt{\alpha^{2}-\gamma \beta^{2}}}\right)-\tanh ^{-1}\left(\frac{\sqrt{\gamma} \beta}{\alpha}\right) \\
& \text { If } \sqrt{\gamma} \beta>|\alpha|, \theta_{C, \text { lim }}=\sinh ^{-1}\left(\frac{1 / 2+\alpha}{\sqrt{\gamma \beta^{2}-\alpha^{2}}}\right)-\tanh ^{-1}\left(\frac{\alpha}{\sqrt{\gamma} \beta}\right) \\
& \text { if } \alpha=\sqrt{\gamma} \beta, \theta_{C, \text { lim }}=\ln \left(\frac{1 / 2+\alpha}{\alpha}\right) \quad \text { (fully symmetric system) }
\end{aligned}
$$

These limiting values represent the half-reaction times for background amplification and they only depend on the parameters $\alpha, \beta$ and $\gamma$. Varying the actual values of $\alpha$ and $\beta$ clearly evidences an increase in $\theta_{\text {C,lim }}$ as $\alpha$ and $\beta$ decrease while $\gamma$ is kept constant (Figure 4). The consequence is an extension of the linear range in the calibration plot, while keeping the analytical sensitivity constant (i.e., a constant slope for the linear part of the calibration plot).

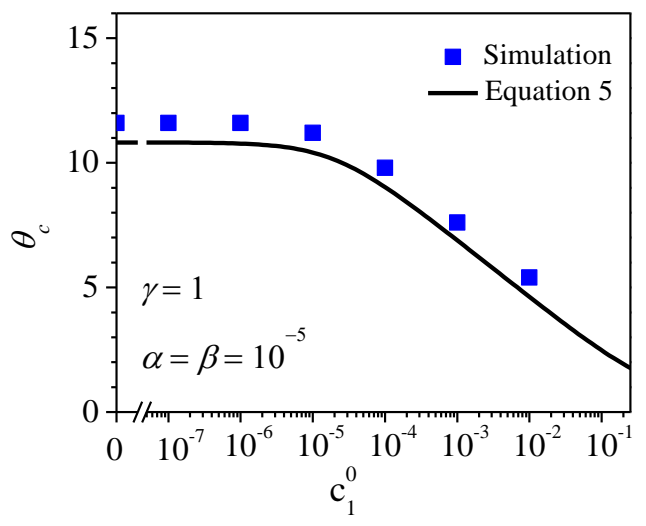

Figure 3. Calibration plot representing the evolution of $\theta_{c}$ as a function of $c_{1}^{0}$. Plain black line: plot of eq. 5. Parameters set: $\alpha=10^{-5}, \beta=10^{-5}, \gamma=1$. Blue squares: $\theta_{c}$ extracted from numerical simulations performed with the COPASI software and using the following set of parameters: $k_{1}=k_{2}=1 \mathrm{M}^{-1} \cdot \mathrm{s}^{-1}, k_{3}=k_{4}=$ $10^{-5} \mathrm{~s}^{-1},\left[\mathrm{C}_{1}\right]_{0}=10^{-3} \mathrm{M},\left[\mathrm{R}_{1}\right]_{0}=\left[\mathrm{R}_{2}\right]_{0}=1 \mathrm{M}$.

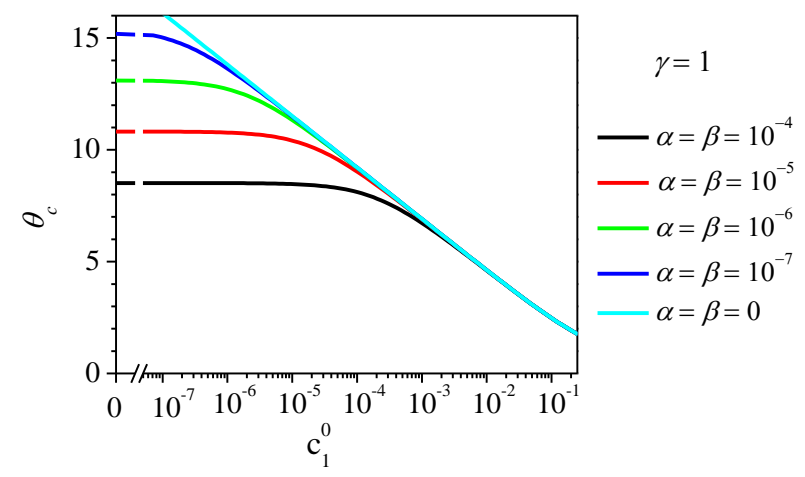

Figure 4. Calibration plots of $\theta_{c}$ as a function of $c_{1}^{0}$ for different values of $\alpha$ and $\beta$. The plots were calculated using eq. 5 .

From an analytical perspective, an important feature that the present model can help anticipate from a given set of specific and nonspecific kinetic parameters is the limit of detection of $c_{1}^{0}$. Given the expression of $c_{1}$ (eq. 1), it is easy to differentiate the specific amplification of $c_{1}\left(c_{1, \mathrm{~s}}\right)$ in response to a triggering concentration $c_{1}^{0}$ from the nonspecific amplification due to the instabilities of reservoirs $\left(c_{1, n s}\right)$ : 
$c_{1}=c_{1, \mathrm{~s}}+c_{1, \mathrm{~ns}}$

with

$c_{1, \mathrm{~s}}=c_{1}^{0} \cosh (\theta)$

$c_{1, \mathrm{~ns}}=\alpha(\cosh (\theta)-1)+\sqrt{\gamma} \beta \sinh (\theta)$

In a first approximation, detection of a specific response is possible as long as $c_{1, \mathrm{~s}}>C_{1, \mathrm{~ns}}$

or $\frac{c_{1, \mathrm{~ns}}}{c_{1, \mathrm{~s}}}=\frac{\alpha}{c_{1}^{0}}\left(\frac{\cosh (\theta)-1}{\cosh (\theta)}\right)+\frac{\sqrt{\gamma} \beta}{c_{1}^{0}} \tanh (\theta)$

Then, $\left(\frac{c_{1, \mathrm{~ns}}}{c_{1, \mathrm{~s}}}\right)_{\theta>>1}=\frac{\alpha+\sqrt{\gamma} \beta}{c_{1}^{0}}$

We can therefore define a concentration limit $c_{1, \min }^{0}$ that fulfills the following requirement:

$\left(\frac{c_{1, \mathrm{~ns}}}{c_{1, \mathrm{~s}}}\right)_{\theta>>1}=1=\frac{\alpha+\sqrt{\gamma} \beta}{c_{1, \min }^{0}}$

which also corresponds to the case where both specific and nonspecific contributions are equal. It follows that

$c_{1, \min }^{0}=\alpha+\sqrt{\gamma} \beta$

This dimensionless equation also corresponds to the following expression:

$\left[\mathrm{C}_{1}\right]_{0, \min }=\frac{k_{3}^{\prime}}{k_{1}^{\prime}}+\frac{k_{4}^{\prime}}{\sqrt{k_{1}^{\prime} k_{2}^{\prime}}}$

Eqs. 8 and 9 , highlights the contributions of each $R_{1}$ and $R_{2}$ instabilities to the detection limit. Indeed, two limiting cases can be defined regarding the relative instability controlling the background response. The principal limitation is either the relative instability of $\mathrm{R}_{2}$ (in that case $\alpha \gg \sqrt{\gamma} \beta$ and $c_{1, \min }^{0}=\alpha$ or $\left.\left[\mathrm{C}_{1}\right]_{0, \text { min }}=\frac{k_{3}^{\prime}}{k_{1}^{\prime}}\right)$, or the relative instability of $\mathrm{R}_{1} \quad(\alpha<<\sqrt{\gamma} \beta$ and $c_{1, \min }^{0}=\sqrt{\gamma} \beta$ or $\left.\left[\mathrm{C}_{1}\right]_{0, \min }=\frac{k_{4}^{\prime}}{\sqrt{k_{1}^{\prime} k_{2}^{\prime}}}\right)$.

The passage from one limiting case to the other can be better appreciated from the parameter $\sigma$.

$\sigma=\frac{\alpha}{\sqrt{\gamma} \beta}=\frac{k_{3}^{\prime}}{k_{4}^{\prime}} \sqrt{\frac{k_{2}^{\prime}}{k_{1}^{\prime}}}$

The first limiting case, i.e. when $\sigma>1$ (control by the relative instability of $R_{2}$ ), allows calculating that for reaching a LOD of 1 $\mathrm{pM}$ in target $\mathrm{C}_{1}$, it is necessary that the relative instability associated with the catalytic loop 1 is at most of the order of 10 12 . This actually gives an idea of the degree of stability required to detect traces of $\mathrm{C}_{1}$. In the second case, i.e. when $\sigma<<1$ (control by the relative instability of $R_{1}$ ), it depends on the kinetic characteristic of both loops 1 and 2, and more specifically on the relative instability associated with loop 2 as well as the kinetic symmetry factor.

The next question worth addressing is how to design a new molecular-based autocatalytic amplification system to improve the LOD of a target? In the first limiting case $(\sigma>>1)$, the detection limit is totally independent of the kinetic characteristic of loop 2 (production of $\mathrm{C}_{1}$ from $\mathrm{R}_{1}$ ). It is therefore useless to design system with enhanced stability of $R_{1}$ and/or reactivity of $\mathrm{C}_{1}$. The only strategy is to independently improve the stability of $\mathrm{R}_{2}$ and/or the reactivity of $\mathrm{C}_{2}$. In the second limiting case $(\sigma<<1)$, several options are possible: improving the stability of $\mathrm{R}_{1}$ and/or increasing the reactivity of either $\mathrm{C}_{1}$ or $\mathrm{C}_{2}$.

(ii) $C_{2}$ is the trigger

Let us now consider the case where the other catalyst $\mathrm{C}_{2}$ is the trigger. Similar expressions than previously can be established with, as initial conditions, $c_{1}^{0}=0$ and $c_{2}^{0} \neq 0$ :

$c_{1}=\alpha(\cosh (\theta)-1)+\sqrt{\gamma}\left(c_{2}^{0}+\beta\right) \sinh (\theta)$

$c_{2}=c_{2}^{0} \cosh (\theta)+\frac{\alpha}{\sqrt{\gamma}} \sinh (\theta)+\beta(\cosh (\theta)-1)$

Half-reaction time is now:

If $\alpha>\left|\sqrt{\gamma}\left(c_{2}^{0}+\beta\right)\right|$,

$\theta_{C}=\cosh ^{-1}\left(\frac{1 / 2+\alpha}{\sqrt{\alpha^{2}-\gamma\left(c_{2}^{0}+\beta\right)^{2}}}\right)-\tanh ^{-1}\left(\frac{\sqrt{\gamma}\left(c_{2}^{0}+\beta\right)}{\alpha}\right)$

If $\sqrt{\gamma}\left(c_{2}^{0}+\beta\right)>|\alpha|$,

$\theta_{C}=\sinh ^{-1}\left(\frac{1 / 2+\alpha}{\sqrt{\gamma\left(c_{2}^{0}+\beta\right)^{2}-\alpha^{2}}}\right)-\tanh ^{-1}\left(\frac{\alpha}{\sqrt{\gamma}\left(c_{2}^{0}+\beta\right)}\right)$

As previously, we may define the smallest concentration of trigger that would lead to a response that differs from the background response:

$c_{2, \min }^{0}=\beta+\frac{\alpha}{\sqrt{\gamma}}=\frac{c_{1, \min }^{0}}{\sqrt{\gamma}}$

or

$\left[\mathrm{C}_{2}\right]_{0, \min }=\frac{k_{4}^{\prime}}{k_{2}^{\prime}}+\frac{k_{3}^{\prime}}{\sqrt{k_{1}^{\prime} k_{2}^{\prime}}}$

And again, it is worth specifying the two limiting cases:

(i) when $\sigma<<1, c_{2, \min }^{0}=\beta$ and $\left[\mathrm{C}_{2}\right]_{0, \min }=\frac{k_{4}^{\prime}}{k_{2}^{\prime}}$. The detection limit in this case is only dependent on the stability of $R_{1} v s$. the reactivity of $\mathrm{C}_{1}$.

(ii) when $\sigma \gg 1, c_{2, \min }^{0}=\frac{\alpha}{\sqrt{\gamma}}$ and $\left[\mathrm{C}_{2}\right]_{0, \min }=\frac{k_{3}^{\prime}}{\sqrt{k_{1}^{\prime} k_{2}^{\prime}}}$. The detection

limit is determined by the stability of $R_{2}$, and the reactivity of both $\mathrm{C}_{1}$ and $\mathrm{C}_{2}$.

Interestingly, the detection limits for each catalyst are related through the symmetry factor $\gamma$.

$\frac{c_{2, \min }^{0}}{c_{1, \min }^{0}}=\frac{1}{\sqrt{\gamma}}$ 
This last expression demonstrates that it is equally efficient to trigger the cross-catalytic process by any of the catalyst when the system is symmetric $(\gamma=1)$. Conversely, if the system is highly dissymmetric, the system is more sensitive to detect one catalyst over the other, that is, either $\mathrm{C}_{1}$ if $\gamma<<1$ or $\mathrm{C}_{2}$ if $\gamma>1$. In other words, the fastest catalyst would be more sensitively detected.

\section{Triggering the system by catalytic production of a cross- catalyst}

From an analytical point of view, it can be interesting not only to detect the autocatalyst itself but also indirectly to detect a chemical event (for instance, a catalytic reaction) that produces the autocatalyst. ${ }^{[19,26,28,29]}$ If the autocatalytic process is of crosscatalysis type, the corresponding situation is "catalysis-to-crosscatalysis", as depicted in Scheme 1. We will thus consider here a case where a cross-catalyst is initially produced by a third catalytic reaction (catalytic loop 0), Cat being the additional catalyst and $k$ the associated first-order kinetic constant. Here, we must consider the two cases where either $C_{1}$ or $C_{2}$ is the product of the additional catalytic reaction.

$\mathrm{S} \underset{\text { Cat }}{\stackrel{k}{\longrightarrow}} \mathrm{C}_{1}$ or $\mathrm{C}_{2} \quad$ (catalytic loop 0)

\section{(i) Catalytic production of $C_{1}$}

The new set of ODEs describing the system is now:

$$
\begin{aligned}
& \frac{d\left[\mathrm{C}_{1}\right]}{d t}=k_{2}^{\prime}\left[\mathrm{C}_{2}\right]+k_{4}^{\prime}+k[\mathrm{Cat}] \\
& \frac{d\left[\mathrm{C}_{2}\right]}{d t}=k_{1}^{\prime}\left[\mathrm{C}_{1}\right]+k_{3}^{\prime}
\end{aligned}
$$

Or, in a dimensionless representation:

$$
\begin{aligned}
& \frac{d^{2} c_{1}}{d \theta^{2}}-c_{1}=\alpha \\
& \frac{d^{2} c_{2}}{d \theta^{2}}-c_{2}=\beta+\kappa c_{\mathrm{Cat}} \\
& \text { with } \kappa=\frac{k}{k_{2}^{\prime}} \text { and } c_{\mathrm{Cat}}=\frac{[\mathrm{Cat}]}{\left[\mathrm{R}_{1}\right]_{0}}
\end{aligned}
$$

$\kappa$ is a dimensionless parameter that represents here the ratio between the production rate of $\mathrm{C}_{1}$ through loop 0 and loop 2 . In that configuration, the trigger being the catalyst Cat itself, the above system of equations must therefore being solved with

$c_{\text {Cat }}^{0} \neq 0, c_{1}^{0}=0$ and $c_{2}^{0}=0$.

It follows that:

$$
\begin{aligned}
& c_{1}=\alpha(\cosh (\theta)-1)+\sqrt{\gamma}\left(\beta+\kappa c_{\text {Cat }}^{0}\right) \sinh (\theta) \\
& c_{2}=\frac{\alpha}{\sqrt{\gamma}} \sinh (\theta)+\left(\beta+\kappa c_{\text {Cat }}^{0}\right)(\cosh (\theta)-1)
\end{aligned}
$$

With the minimum concentration of Cat that can be detected being given by:
$c_{\mathrm{Cat}, \min }^{0}=\frac{\frac{\alpha}{\sqrt{\gamma}}+\beta}{\kappa}$

It follows that

$\frac{c_{2, \min }^{0}}{c_{\mathrm{Cat}, \min }^{0}}=\kappa$

Cat and $\mathrm{C}_{2}$ are both catalysts of the production of $\mathrm{C}_{1}$, their respective detection limits are thus related only through the single parameter $\kappa$, and strategies to improve the detection of Cat are thus similar to the ones improving the detection of $\mathrm{C}_{2}$. One may also conclude that the best catalyst will be the one more sensitively detected. More interestingly, this help rationalizing the gain in detection limit expected for a given catalyst. For instance, if the LOD of $\mathrm{C}_{2}$ is in the pM range, then the LOD of Cat can be expected in the $\mathrm{fM}$ range if $\mathrm{C}_{1}$ is produced 1000 times more rapidly through loop 0 than through loop 2.

The concentration of the reservoir $\mathrm{R}_{1}$ is still $r_{1}=1-c_{1}$ if the reactant of the additional catalytic reaction is a substrate $S$. In the case the substrate is $R_{1}$ itself, then a supplementary term is expected, i.e. $r_{1}=1-c_{1}-\sqrt{\gamma} \kappa c_{\mathrm{Cat}} \theta$. This last term may be neglected as soon as $\theta>>1$, so that in both cases, the halfreaction time is:

for $\alpha>\left|\sqrt{\gamma}\left(\beta+\kappa c_{\text {Cat }}\right)\right|$,

$\theta_{C}=\cosh ^{-1}\left(\frac{1 / 2+\alpha}{\sqrt{(\alpha)^{2}-\gamma\left(\beta+\kappa c_{\mathrm{Cat}}\right)^{2}}}\right)-\tanh ^{-1}\left(\frac{\sqrt{\gamma}\left(\beta+\kappa c_{\mathrm{Cat}}\right)}{\alpha}\right)$

for $\sqrt{\gamma}\left(\beta+\kappa c_{\text {Cat }}\right)>|\alpha|$,

$\theta_{C}=\sinh ^{-1}\left(\frac{1 / 2+\alpha}{\sqrt{\gamma\left(\beta+\kappa c_{\mathrm{Cat}}\right)^{2}-(\alpha)^{2}}}\right)-\tanh ^{-1}\left(\frac{\alpha}{\sqrt{\gamma}\left(\beta+\kappa c_{\mathrm{Cat}}\right)}\right)$

The above equations are close to the one obtained in the previous section, so that calibration plots (half-reaction time vs. $\left.[\mathrm{Cat}]_{0}\right)$ of similar shapes can be predicted.

In order to go towards an even more sensitive system, one can of course imagine a two-step procedure where $C_{1}$ is preaccumulated during $t_{\mathrm{p}}$ through loop 0 before adding the other reservoirs. In that case, the above system of equations must therefore being solved with

$c_{1}^{0}=\frac{k[\mathrm{Cat}] t_{p}}{\left[\mathrm{R}_{1}\right]_{0}}=\kappa c_{\mathrm{Cat}} \theta_{p}$ and $c_{2}^{0}=0$ (with $\left.\theta_{p}=k_{2}^{\prime} t_{p}\right)$.

It follows:

$c_{1}=\left(\kappa c_{\mathrm{Cat}} \theta_{p}\right) \cosh (\theta)+\alpha(\cosh (\theta)-1)+\sqrt{\gamma}\left(\beta+\kappa c_{\mathrm{Cat}}\right) \sinh (\theta)$

and 


$$
c_{\mathrm{Cat}, \min }^{0}=\frac{\alpha+\sqrt{\gamma} \beta}{\kappa\left(\sqrt{\gamma}+\theta_{p}\right)}=\frac{\frac{\alpha}{\sqrt{\gamma}}+\beta}{\kappa\left(1+\frac{\theta_{p}}{\sqrt{\gamma}}\right)}
$$

Evidently, the greater the pre-incubation time, the lower the detection limit. This configuration is particularly interesting when $\frac{\theta_{p}}{\sqrt{\gamma}}>>1$

or for

$t_{p}>>\frac{1}{\sqrt{k_{2}^{\prime} k_{1}^{\prime}}}=t_{s}$

In other words, the higher the autocatalytic efficiency, the more interesting the two-step strategy. The reason is that preaccumulation allows the specific accumulation of $\mathrm{C}_{1}$ and more $\mathrm{C}_{1}$ must be accumulated during the pre-incubation than during the time of a single "autocatalytic turnover". Of course, this is not true if loop 0 involve $R_{1}$ as reactant since $C_{1}$ can also accumulate through $R_{1}$ instability. In that later case, the initial conditions are $c_{1}^{0}=\frac{\left(k[\mathrm{Cat}]+k_{4}^{\prime}\right) t_{p}}{\left[\mathrm{R}_{1}\right]_{0}}=\left(\kappa c_{\mathrm{Cat}}+\beta\right) \theta_{p}$ and $c_{2}^{0}=0$.

(ii) Catalytic production of $C_{2}$

In the case of catalytic production of $\mathrm{C}_{2}$, the set of dimensionless ODEs writes:

$$
\begin{aligned}
& \frac{d^{2} c_{1}}{d \theta^{2}}-c_{1}=\alpha+\kappa c_{\mathrm{Cat}} \\
& \frac{d^{2} c_{2}}{d \theta^{2}}-c_{2}=\beta \\
& \text { with } \kappa=\frac{k}{k_{1}^{\prime}} \text { and } c_{\text {Cat }}=\frac{[\mathrm{Cat}]}{\left[\mathrm{R}_{1}\right]_{0}}
\end{aligned}
$$

After integration with

$c_{\mathrm{Cat}}^{0} \neq 0, c_{1}^{0}=0$ and $c_{2}^{0}=0$, it results:

$c_{1}=\left(\alpha+\kappa c_{\text {Cat }}\right)(\cosh (\theta)-1)+\sqrt{\gamma} \beta \sinh (\theta)$

$c_{2}=\left(\alpha+\kappa c_{\mathrm{Cat}}\right) \sinh (\theta)+\sqrt{\gamma} \beta(\cosh (\theta)-1)$

and

$c_{\mathrm{Cat}, \min }^{0}=\frac{\alpha+\sqrt{\gamma} \beta}{\kappa}=\frac{c_{1, \text { min }}^{0}}{\kappa}=\frac{\sqrt{\gamma} c_{2, \text { min }}^{0}}{\kappa}$

Half-reaction time is now:

if $\kappa c_{\mathrm{Cat}}+\alpha>|\sqrt{\gamma} \beta|$,

$\theta_{C}=\cosh ^{-1}\left(\frac{1 / 2+\alpha}{\sqrt{\left(\kappa c_{\mathrm{Cat}}+\alpha\right)^{2}-\gamma \beta^{2}}}\right)-\tanh ^{-1}\left(\frac{\sqrt{\gamma} \beta}{\kappa c_{\mathrm{Cat}}+\alpha}\right)$ if $\sqrt{\gamma} \beta>\left|\kappa c_{\text {Cat }}+\alpha\right|$,

$\theta_{C}=\sinh ^{-1}\left(\frac{1 / 2+\alpha}{\sqrt{\gamma \beta^{2}-\left(\kappa c_{\mathrm{Cat}}+\alpha\right)^{2}}}\right)-\tanh ^{-1}\left(\frac{\kappa c_{\mathrm{Cat}}+\alpha}{\sqrt{\gamma} \beta}\right)$

\section{Application to an experimental system}

In this last section we aim at validating the above model in the context of our previously reported autocatalytic system based on cross-catalysis. ${ }^{[2]}$ The system consists of two catalytic loops (Scheme 2):

(I) a $\mathrm{H}_{2} \mathrm{O}_{2}$-mediated catalytic deprotection of a masked naphthohydroquinone probe $(P)$ into a naphthoquinone (NQ) in the presence of $\mathrm{O}_{2}$ (catalytic loop 1):

$\mathrm{P}+\mathrm{O}_{2} \underset{\mathrm{H}_{2} \mathrm{O}_{2}}{\stackrel{k^{\prime}}{\longrightarrow}} \mathrm{NQ}+\mathrm{P}^{\prime}$

(ii) a naphthoquinone-based catalytic $\mathrm{H}_{2} \mathrm{O}_{2}$ production through redox cycling in the presence of a reductant (Red) and $\mathrm{O}_{2}$ as the oxidant (catalytic loop 2)

$\mathrm{Red}+\mathrm{O}_{2} \underset{\mathrm{NQ}}{\stackrel{k_{2}^{\prime}}{\longrightarrow}} \mathrm{H}_{2} \mathrm{O}_{2}+\mathrm{Ox}$

The two cross-catalysts $\mathrm{C}_{1}$ and $\mathrm{C}_{2}$ are therefore $\mathrm{H}_{2} \mathrm{O}_{2}$ and $\mathrm{NQ}$, respectively.
A<smiles>[R]c1c([R])c(O)c2ccccc2c1O</smiles>

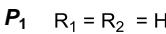
$\begin{array}{lll}P_{2} & R_{1} & =R_{2}=C l\end{array}$

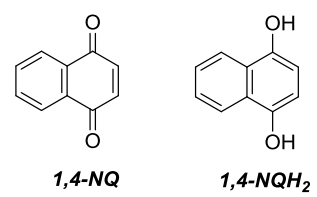<smiles>O=C1C(Cl)=C(Cl)C(=O)c2ccccc21</smiles>

2,3-diCl-1,4-NQ
B

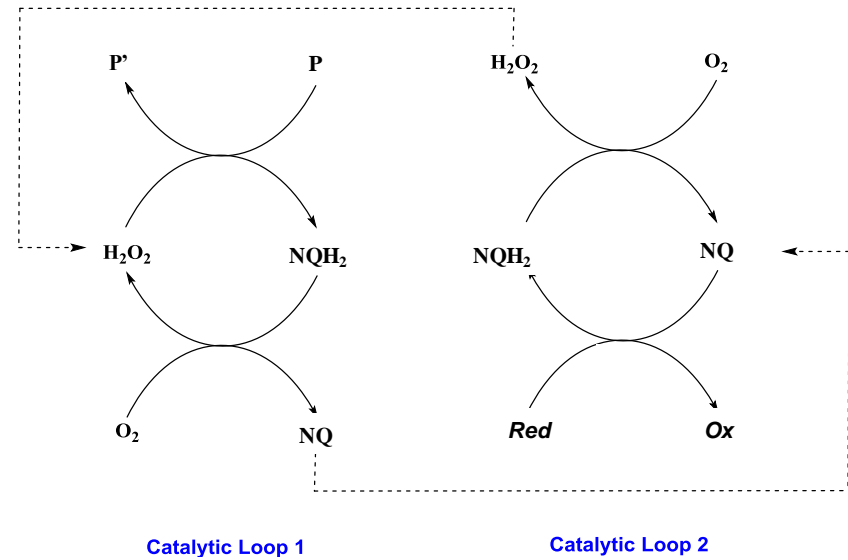

Scheme 2. (A) Chemical structures of the masked naphthoquinone probes $P_{1}$ and $P_{2}$ with the associated naphthoquinones 1,4-NQ and 2,3-diCl-1,4-NQ respectively. The reduced form of 1,4-NQ, i.e. 1,4-NQH $\mathrm{NQ}_{2}$, is also presented. (B) Cross-catalytic reaction scheme showing both catalytic loop 1 and 2. 
Different naphthohydroquinone-based probes protected by ester boronate groups $\left(P_{1}\right.$ and $\left.P_{2}\right)$ as well as chemical or enzyme/substrate reductants (i.e., ascorbate, or PQQ-glucose dehydrogenase/glucose and diaphorase/NADH) were investigated. ${ }^{[28]}$ Reaction progress was monitored by UV-vis spectrophotometry but also electrochemically. ${ }^{[28]}$
The detection of either $\mathrm{H}_{2} \mathrm{O}_{2}$ or redox cycling compounds such as the 2,3-dichloro-naphthoquinone at the level of submicromolar or nanomolar was demonstrated. ${ }^{[28]}$ It was also experimentally shown that these analytical performances were limited by the intrinsic instability of the probe itself.
A

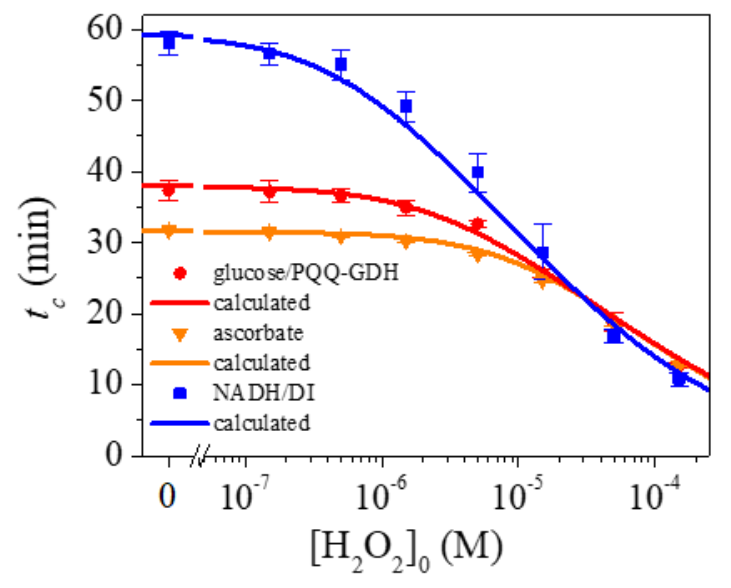

B

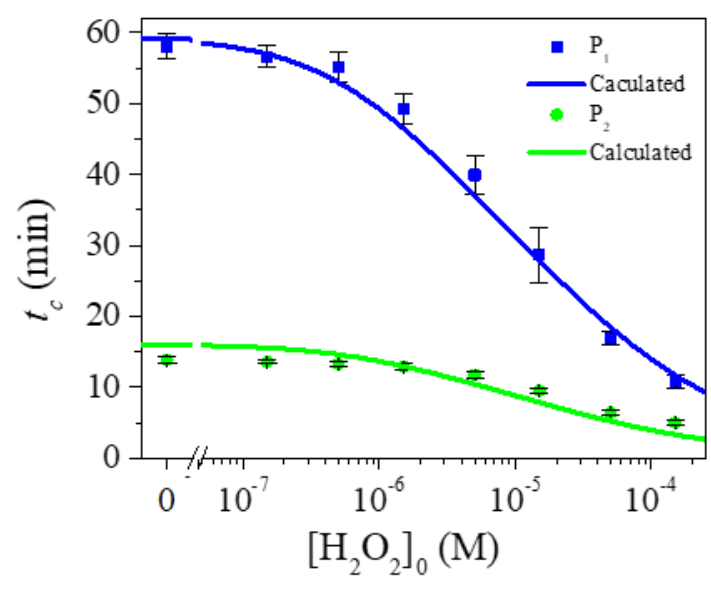

Figure 5. (A, B) Semi-logarithmic calibration plots obtained by reporting the experimental half-reaction times $\left(t_{c}\right)$ extracted from the UV-Vis kinetic traces recorded in a 96-well microtiter plate as a function of different $\mathrm{H}_{2} \mathrm{O}_{2}$ concentrations. All experiments were conducted in an air-saturated $0.1 \mathrm{M}$ Tris buffer ( $\mathrm{pH} 8.5$ ) containing $10 \mu \mathrm{M}$ EDTA, $50 \mu \mathrm{M} \mathrm{P}$, either (orange) $0.5 \mathrm{mM}$ ascorbate, (blue) $10 \mathrm{nM}$ diaphorase and $250 \mu \mathrm{M} \mathrm{NADH}$, or (red) $10 \mathrm{nM}$ PQQ-glucose dehydrogenase and $1 \mathrm{mM}$ glucose, and different $\mathrm{H}_{2} \mathrm{O}_{2}$ concentrations (ranging from 0 to $150 \mu \mathrm{M}$ ). Errors bars are standard deviations from triplicates. Solid lines represent the plots of eq. 5 using the parameters listed in Table 1. (B) Same experimental conditions than in A but with $50 \mu \mathrm{M}$ of (blue) $\mathrm{P}_{1}$ or (green) $\mathrm{P}_{2}$, and $10 \mathrm{nM}$ dipahorase and $250 \mu \mathrm{M}$ $\mathrm{NADH}$. Errors bars are standard deviations from triplicates. Solid lines represent the plots of eq. 5 using the parameters listed in Table 1.

Table 1. Rate constants and related parameters.

\begin{tabular}{|c|c|c|c|c|}
\hline $\begin{array}{l}\text { Probe } \\
\text { Red }\end{array}$ & $\begin{array}{c}\mathrm{P}_{1} \\
\text { Ascorbate }\end{array}$ & $\begin{array}{c}P_{1} \\
\text { PQQ-GDH/Glucose } 3\end{array}$ & $\begin{array}{c}P_{1} \\
\text { Diaphorase/NADH }\end{array}$ & $\begin{array}{c}\mathbf{P}_{2} \\
\text { Diaphorase/NADH }\end{array}$ \\
\hline$k_{1}^{\prime}\left(\mathrm{s}^{-1}\right)$ & $7.5 \times 10^{-5[a] /} / 1.5 \times 10^{-4[b]}$ & $7.5 \times 10^{-5[a]}$ & $7.5 \times 10^{-5}[\mathrm{a}]$ & $7.5 \times 10^{-5[a]}$ \\
\hline$k_{2}^{\prime}\left(\mathrm{s}^{-1}\right)$ & $0.05^{[a]} / 0.05^{[b]}$ & $0.09^{[a]}$ & $0.04^{[a]}$ & $0.5^{\text {[a] }}$ \\
\hline$k_{3}^{\prime}\left(\mathrm{M} \cdot \mathrm{s}^{-1}\right)$ & $6 \times 10^{-10[a] / 5 \times 10^{-10[b]}}$ & $2 \times 10^{-10}$ & $3.5 \times 10^{-11}$ & $5 \times 10^{-11}$ \\
\hline$k_{4}^{\prime}\left(\mathrm{M} \cdot \mathrm{s}^{-1}\right)$ & $10^{-8[a] / 10^{-8[b]}}$ & $<10^{-10}$ & $<10^{-10}$ & $<10^{-10}$ \\
\hline$\sigma$ & $1.55^{[c]}$ & $>70$ & $>8$ & $>33$ \\
\hline$\left[\mathrm{C}_{1}\right]_{0, \min }(\mu \mathrm{M})$ & $13^{[d]}$ & 2.7 & 0.5 & 0.5 \\
\hline
\end{tabular}

${ }^{[a]}$ Estimated from the fit of the calibration plot to the dimensionless form of eqs. 5 and $6 .{ }^{[b]}$ Measured values taken from ref [25]. ${ }^{\left[{ }^{[c]}\right.}$ Calculated from eq. 10.

${ }^{[d]}$ Calculated from eq. 9.

In order to apply the above model, rates of both catalytic loops 1 and 2 were assumed to follow a simple bimolecular reaction, which under large concentration excess of probe and $\mathrm{O}_{2}$ (or Red, depending on the rate determining step of the redox cycling) are expected to be first order towards each of the catalyst. As a result, we can write for the rate of each catalytic loop:

$v_{1}=k_{1}^{\prime}\left[\mathrm{H}_{2} \mathrm{O}_{2}\right]$ and $v_{2}=k_{2}^{\prime}[\mathrm{NQ}]$
For the catalytic loop 1, the reaction of probe deprotection by $\mathrm{H}_{2} \mathrm{O}_{2}$ is indeed the rate determining step as the autoxidation of $\mathrm{NQH}_{2}$ is relatively much faster. The pseudo-first-order rate constant $k_{1}^{\prime}$ is related to the initial probe concentration $[\mathrm{P}]_{0}$ through $k_{1}$, the second-order rate constant for the probe deprotection by $\mathrm{H}_{2} \mathrm{O}_{2}$ (i.e., $k_{1}^{\prime}=k_{1}[\mathrm{P}]_{0}$ ). For the catalytic loop 2, kinetics of the two-electron two-proton redox cycling is quite complex for either chemical or enzymatic reduction of the 
quinone ${ }^{[28]}$ Here, $k_{2}^{\prime}$ represents an apparent global redox cycling rate constant under given experimental conditions. Note that in this example the two loops actually share a common reaction step, i.e. the autoxidation of the naphthohydroquinone. However, they are kinetically independent since this common step is not simultaneously the rate determining step of both loops. If this were the case, we could still treat it with the same model with an equality between both apparent global first-order rate constant leading to symmetry factor $\gamma$ equal to 1 . Finally, to complete the reaction scheme and account for the background amplification, two additional reactions should be considered:

$$
\mathrm{P}+\mathrm{O}_{2} \stackrel{k_{3}^{\prime}}{\longrightarrow} \mathrm{NQ}
$$

and

$$
\mathrm{Red}+\mathrm{O}_{2} \stackrel{k_{4}^{\prime}}{\longrightarrow} \mathrm{H}_{2} \mathrm{O}_{2}+\mathrm{Ox}
$$

They correspond respectively to the self-degradation of the probe due to the instability of the spacer arm that eventually leads to $\mathrm{NQ}$ in the presence of $\mathrm{O}_{2}$, and to the autoxidation of the reducing agent.

Figure 5 shows the calibration plot derived from the experimental results using $P_{1}$ and each of the three different reducing agents (Figure $5 A$ ) or using the two different probes $P_{1}$ and $\mathrm{P}_{2}$ and the same reducing diaphorase/NADH system (Figure $5 \mathrm{~B}$ ). Here, $\mathrm{H}_{2} \mathrm{O}_{2}$ (i.e., $\mathrm{C}_{1}$ ) is the trigger of the cross-catalytic reaction. The plots were therefore fitted with dimensional forms of eqs. 5 and 6 and using the parameters gathered in Table 1. Considering the results with $\mathrm{P}_{1}$ (Figure $5 \mathrm{~A}$ ), the corresponding values of the different $k$ 's agree quite well with those experimentally determined in the case of ascorbate as the reducing agent, validating thus the model. In this case the value of $\sigma$ is 1.55 , therefore the background amplification is indeed controlled mostly by the probe's instability, even though the ascorbate autoxidation significantly contributes. Glucose and $\mathrm{NADH}$ are much less prone to autoxidation than ascorbate and therefore the values of $\sigma$ are expectedly increased, demonstrating that the main source of background amplification is now almost exclusively linked to the probe instability. The 2,3dichloro-1,4-naphthoquinone released from $\mathrm{P}_{2}$ is a better redox cycler than the 1,4-NQ released from $P_{1}$ when using the diaphorase/NADH reducing agent. ${ }^{[28]}$ This is confirmed here by the greater value of $k_{2}^{\prime}$ (almost 20-fold higher) extracted from the fit in Figure 5B. A surprising observation is the dependence of the probe's instability rate $\left(k_{3}^{\prime}\right)$ with the nature of the reducing agent. This apparent behavior seems to correlate with the reducing power (diaphorase/NADH $>\mathrm{PQQ}$-glucose dehydrogenase/glucose $>$ ascorbate). Indeed, the higher the reducing power of reductant, the slower the apparent degradation of the probe. This might be explained by a slower apparent autoxidation of $\mathrm{NQH}_{2}$ under increasing reducing conditions, as the contribution to the autoxidation of the comproportionation reaction with $\mathrm{NQ}$ is expected to vanish. ${ }^{[30]}$ Actually, this is what is observed for the redox cycling with ascorbate when using increasing concentrations of ascorbate (see SI of [28]).

In terms of detection limit, extracted values allow the estimation of $\left[\mathrm{C}_{1}\right]_{0, \min }$ through eq. 9. Results are presented in Table 1 and satisfactorily account for the experimental one. In particular, the reducing couple diaphorase/NADH provides the best analytical performances.

Even if not done experimentally, we can similarly estimate the detection limit when 1,4-NQ is the trigger. We established that $c_{2, \min }^{0}=c_{1, \min }^{0} / \sqrt{\gamma}$, which leads to a detection limit in 1,4-NQ of ca.
$20 \mathrm{nM}$ in the case of using the diaphorase/NADH reducing partner. However, Figure 6 shows the calibration plot obtained from an experiment where the 2,3-diCl-1,4-NQ, instead of 1,4$N Q$, is used as a trigger with $P_{1}$ and diahorase/NADH reservoirs. This is a typical example of catalysis-to-cross-catalysis where 2,3-diCl-1,4-NQ is the catalyst of loop 0 , i.e. the production of $\mathrm{H}_{2} \mathrm{O}_{2}$ in the presence of diaphorase/NADH and $\mathrm{O}_{2}$ (catalytic production of the cross-catalyst $\mathrm{C}_{1}$ from $\mathrm{R}_{1}$ ). Taking into account the values of apparent redox cycling rate for both 2,3-diCl-1,4$\mathrm{NQ}$ and 1,4-NQ (i.e., $k=k_{2, \mathrm{P}_{2}}^{\prime}=0.5 \mathrm{~s}^{-1}$ and $k_{2, \mathrm{P}_{1}}^{\prime}=0.04 \mathrm{~s}^{-1}$, respectively), the value of $\kappa$ can be estimated to 12.5 . Therefore, the calibration curve can be calculated from eqs. 20 (red curve in Figure 6) and 21 (black curve in Figure 6) taking into account all pre-evaluated constants in Table 1 . It results in a particularly good agreement with the experimental data, confirming the validity and utility of the catalysis-to-cross-catalysis model. In terms of analytical performances, a LOD of $1.6 \mathrm{nM}$ 2,3-dichloro1,4-naphthoquinone can be calculated from eq. 17 , a value that agrees quite well to the one we had previously determined experimentally $(\sim 0.5 \mathrm{nM}){ }^{[28]}$

Finally, with the aim of improving analytical performances, the design of a better system may be guided by the model. From Table 1 , the fact that $\sigma$ is always greater than 1 means that background amplification is controlled by the relative instability of the probe. In terms of detection limit, this respectively means from eqs. 5,16 and 20 that:

$$
\begin{aligned}
& {\left[\mathrm{C}_{1}\right]_{0, \text { min }}=\left[\mathrm{H}_{2} \mathrm{O}_{2}\right]_{0, \text { min }}=\frac{k_{3}^{\prime}}{k_{1}^{\prime}}} \\
& {\left[\mathrm{C}_{2}\right]_{0, \text { min }}=[1,4-\mathrm{NQ}]_{0, \text { min }}=\frac{k_{3}^{\prime}}{\sqrt{k_{1}^{\prime} k_{2}^{\prime}}}} \\
& {[\mathrm{Cat}]_{0, \text { min }}=[2,3-\mathrm{diCl}-1,4-\mathrm{NQ}]_{0, \text { min }}=\sqrt{\frac{k_{2}^{\prime}}{k_{1}^{\prime}}} \frac{k_{3}^{\prime}}{k}}
\end{aligned}
$$

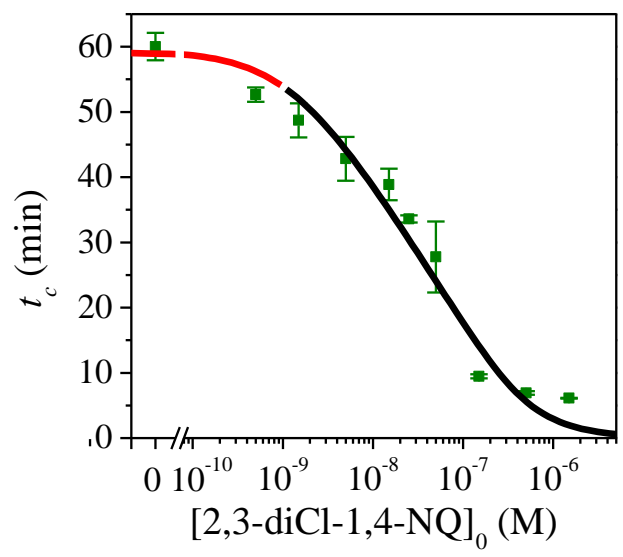

Figure 6. Semi-logarithmic calibration plot obtained by reporting the experimental half-reaction times extracted from UV-Vis kinetic traces at 340 $\mathrm{nm}$ (monitoring of NADH consumption) in a 96-well microtiter plate as a function of different 2,3-dichloro-1,4-NQ concentrations (here represented by $\left.[N Q]_{0}\right)$. All experiments were conducted in an air-saturated $0.1 \mathrm{M}$ Tris buffer (pH 8.5) containing $10 \mu \mathrm{M}$ EDTA, $50 \mu \mathrm{M} \mathrm{P}_{1}, 10 \mathrm{nM}$ diaphorase, $250 \mu \mathrm{M}$ NADH, and the different concentrations of 2,3-dichloro-1,4-NQ (ranging from 0 to $1500 \mu \mathrm{M})$. Errors bars are standard deviations from triplicates. Solid lines represent the plots of eqs. 20 (red) and 21 (black) using the parameters listed in Table 1. 
It is therefore straightforward that designing a more stable probe is beneficial in all three cases (decrease of $k_{3}^{\prime}$ while keeping all other constants the same). Similarly, the reactivity of the probe toward $\mathrm{H}_{2} \mathrm{O}_{2}\left(k_{1}^{\prime}\right)$ can be advantageously fasten, but the gain would be greater for detecting $\mathrm{H}_{2} \mathrm{O}_{2}$ than the NQs. At last, a probe releasing a better redox cycler ( $\mathrm{NQ}$ with increased $k_{2}^{\prime}$ ) would not affect the propensity to detect $\mathrm{H}_{2} \mathrm{O}_{2}$ sensitively (as seen in Table 1 comparing $\mathrm{P}_{1}$ and $\mathrm{P}_{2}$ ), and would even be detrimental to detect 2,3-diCl-1,4-NQ.

\section{Conclusion}

Autocatalytic processes such as those based on cross-catalysis surely represent a powerful route towards the development of efficient molecular-based signal amplifications. However, background amplification due to intrinsic instability of the coreactants can limit the associated performances and prevent to take fully advantage of such amplification rates. Here we developed a simple kinetic model of cross-catalysis with build-in background amplification in order to decipher specific from nonspecific contributions. Thus, we underlined the key parameters governing the response of the system and discussed the analytical performances that can be expected from a given set of kinetic parameters. In particular, the detection limit has been demonstrated to be directly related to the relative instability within each catalytic loop (ratios of the uncatalyzed to catalyzed reaction rates) and to the kinetic symmetry factor (ratio of the catalytic rate of each loop). Does increasing the rate of one of the two loops necessarily permit greater analytical performances? We learned in fact that increasing reactivity (acceleration of one of the two loops) always accelerate both specific and non-specific responses (as $t_{s}=1 / \sqrt{k_{1}^{\prime} k_{2}^{\prime}}$ decrease) but those two can be better discriminated only in specific cases, as demonstrated with eqs 9 or $16:(I)$ in the case the accelerated loop is the one catalyzed by the trigger, the effect (gain in discrimination) is proportional to the kinetic gain if the background amplification is controlled by the relative instability of the same loop and only proportional to its square root otherwise; (ii) in the case the accelerated loop is the one catalyzed by the non-triggering cross-catalyst; there is an effect only if the background amplification is controlled by the relative instability of the same loop and the gain in discrimination is proportional to the square root of the kinetic gain. We also considered the case of catalysis-to-cross-catalysis in which one of the cross-catalyst is catalytically produced though an independent reaction, either simultaneously or during a preaccumulation step. The different models were validated with a previously reported experimental dataset. We believe that this model, while helping to rationalize the analytical performances, can also serve as a guide for the improvement and development of such systems.

\section{Experimental Section}

The experimental data used here are taken from Ref. [25].
We would like to posthumously thank Prof. Jean-Michel Savéant for everything he taught us. We hope that the present work reflects how he influenced our way to tackle scientific issues by using adequate models to shed the light on the most important physico-chemical parameters that governs dynamics and reactivity in complex phenomena. This work was supported by the Agence Nationale pour la Recherche (ANR ECOSENS project ANR - 19 - CE29 - 0022 - 0).

\section{Keywords: Autocatalysis • Cross-catalysis • Molecular} amplification •

[1] P. Scrimin, L. J. Prins, Chem. Soc. Rev. 2011, 40, 4488-4505.

[2] S. Goggins, C. G. Frost, Analyst 2016, 141, 3157-3218.

[3] S. Zhang, A. Garcia-D’Angeli, J. P. Brennan, Q. Huo, Analyst 2014, 139, 439-445.

[4] R. M. Lequin, Clinical Chemistry 2005, 51, 2415-2418.

[5] E. Valero, R. Varón, F. García-Carmona, Biophys. J. 2004, 86, 35983606 .

[6] Z. Gao, L. Hou, M. Xu, D. Tang, Scientific Reports 2014, 4, 3966.

[7] T. Zauner, R. Berger-Hoffmann, K. Müller, R. Hoffmann, T. Zuchner, Anal. Chem. 2011, 83, 7356-7363.

[8] A. J. Bissette, S. P. Fletcher, Angewandte Chemie International Edition 2013, 52, 12800-12826.

[9] A. I. Hanopolskyi, V. A. Smaliak, A. I. Novichkov, S. N. Semenov, ChemSystemsChem 2021, 3, e2000026.

[10] A. Blokhuis, D. Lacoste, P. Nghe, PNAS 2020, 117, 25230-25236.

[11] R. Plasson, A. Brandenburg, L. Jullien, H. Bersini, J. Phys. Chem. A 2011, 115, 8073-8085

[12] W. Hordijk, Journal of Theoretical Biology 2017, 435, 22-28.

[13] A. Horváth, Physical Chemistry Chemical Physics 2021, 23, 7178-7189.

[14] C. N. Hinshelwood, J. Chem. Soc. 1952, 745-755

[15] J. Wilhelm, A. Pingoud, ChemBioChem 2003, 4, 1120-1128.

[16] J. V. Ness, L. K. V. Ness, D. J. Galas, PNAS 2003, 100, 4504-4509.

[17] M. S. Reid, R. E. Paliwoda, H. Zhang, X. C. Le, Anal. Chem. 2018, 90 11033-11039.

[18] G. Urtel, M. Van Der Hofstadt, J.-C. Galas, A. Estevez-Torres, Biochemistry 2019, 58, 2675-2681.

[19] G. Gines, R. Menezes, K. Nara, A.-S. Kirstetter, V. Taly, Y. Rondelez, Sci. Adv. 2020, 6, eaay5952.

[20] K. Shi, S. Xie, R. Tian, S. Wang, Q. Lu, D. Gao, C. Lei, H. Zhu, Z. Nie, Sci. Adv. 2021, 7, eabc7802.

[21] T. Sano, C. L. Smith, C. R. Cantor, Science 1992, 258, 120-122.

[22] D. Y. Ryazantsev, D. V. Voronina, S. K. Zavriev, Biochemistry Moscow 2016, 81, 1754-1770.

[23] H. Liu, L. Zhang, Y. Xu, J. Chen, Y. Wang, Q. Huang, X. Chen, Y. Liu Z. Dai, X. Zou, Z. Li, Talanta 2019, 204, 248-254.

[24] D. Mao, T. Chen, H. Chen, M. Zhou, X. Zhai, G. Chen, X. Zhu, Analyst 2019, 144, 4060-4065.

[25] E. Sella, D. Shabat, J. Am. Chem. Soc. 2009, 131, 9934-9936.

[26] K. Yeung, K. M. Schmid, S. T. Phillips, Chem. Commun. 2012, 49, 394-396.

[27] X. Sun, D. Shabat, S. T. Phillips, E. V. Anslyn, Journal of Physical Organic Chemistry 2018, 31, e3827.

[28] J. Pallu, C. Rabin, G. Creste, M. Branca, F. Mavré, B. Limoges,

Chemistry - A European Journal 2019, 25, 7534-7546.

[29] J. P. Goertz, I. M. White, Angewandte Chemie International Edition

2017, 56, 13411-13415.

[30] R. Munday, Redox Report 1997, 3, 189-196.

\section{Acknowledgements}


